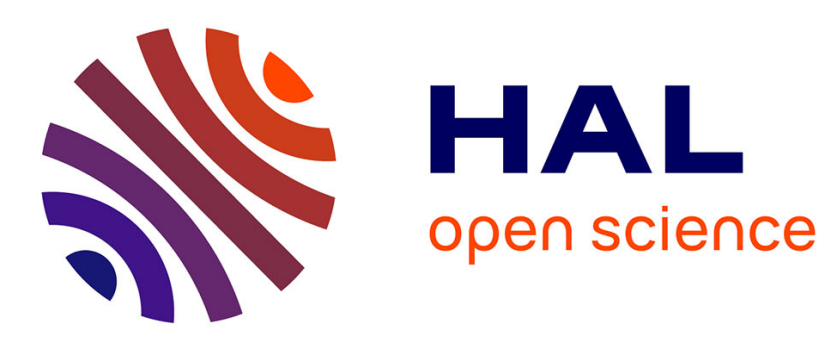

\title{
Scanning Electron Microscope Calibration Using a Multi-Image Non-Linear Minimization Process
}

\author{
Le Cui, Eric Marchand
}

\section{To cite this version:}

Le Cui, Eric Marchand. Scanning Electron Microscope Calibration Using a Multi-Image NonLinear Minimization Process. International Journal of Optomechatronics, 2015, 9 (2), pp.151-169. 10.1080/15599612.2015.1034903 . hal-01241405

\section{HAL Id: hal-01241405 \\ https://hal.inria.fr/hal-01241405}

Submitted on 10 Dec 2015

HAL is a multi-disciplinary open access archive for the deposit and dissemination of scientific research documents, whether they are published or not. The documents may come from teaching and research institutions in France or abroad, or from public or private research centers.
L'archive ouverte pluridisciplinaire HAL, est destinée au dépôt et à la diffusion de documents scientifiques de niveau recherche, publiés ou non, émanant des établissements d'enseignement et de recherche français ou étrangers, des laboratoires publics ou privés. 


\title{
Scanning electron microscope
}

\author{
calibration using a multi-image
}

non-linear minimization process

\begin{abstract}
A scanning electron microscope (SEM) calibrating approach based on non-linear minimization procedure is presented in this article $^{1}$. Both the intrinsic parameters and the extrinsic parameters estimations are achieved simultaneously by minimizing the registration error. The proposed approach considers multi-images of a multi-scale calibration pattern view from different positions and orientations. Since the projection geometry of the scanning electron microscope is different from that of a classical optical sensor, the perspective projection model and the parallel projection model are considered and compared with distortion models. Experiments are realized by varying the position and

\footnotetext{
${ }^{1} \mathrm{~A}$ part of this article has been published in IEEE International Conference on Robotics and Automation (ICRA), 2014
} 
the orientation of a multi-scale chessboard calibration pattern from $300 \times$ to $10,000 \times$. The experimental results show the efficiency and the accuracy of this approach.

\begin{tabular}{|c|c|c|c|}
\hline \multicolumn{4}{|c|}{ NOMENCLATURE } \\
\hline$\delta_{u}, \delta_{v}$ & radial distortion coefficients & & projection of a point on im- \\
\hline$\gamma$ & skewness coefficients & & age plane \\
\hline$\lambda$ & proportional coefficient & $\mathbf{x}_{p}$ & coordinates on pixel of the \\
\hline$\mu$ & coefficient in Levenberg- & & projection of a point on im- \\
\hline & Marquardt method & & age plane \\
\hline e & error between current and & $\xi$ & intrinsic parameters \\
\hline & desired image feature & ${ }^{c} \mathbf{X}$ & coordinates of a point in sen- \\
\hline $\mathbf{I}$ & identity matrix & & sor frame \\
\hline $\mathbf{J}_{p}$ & image Jacobian & $p_{x}, p_{y}$ & pixel/meter ratio \\
\hline $\mathbf{r}$ & extrinsic parameters (pose) & $s_{1}, s_{2}$ & spiral coefficients \\
\hline $\mathbf{V}$ & velocity & $u_{0}, v_{0}$ & coordinates \\
\hline $\mathbf{X}$ & coordinates on meter of the & & point \\
\hline
\end{tabular}




\section{Introduction}

Scanning electron microscope (SEM) is an electron microscope where a focused beam of electrons is used to scan the surface of a specimen. This is an essential instrument to display, measure and manipulate the micro and nano-structure with a micrometers or nanometers accuracy. When the task requires the computation of metric information from the acquired twodimensional (2D) images, the calibration of the SEM is an important issue to be considered.

Calibration of an optical sensor has been widely investigated over the last decades. The goal of the calibration process is to determine the set of parameters which define the relationship between the three-dimensional (3D) coordinates of an object point on the observed specimen and its projection in the image plane (such parameters that include, in an optical system, the focal length, the dimension of pixel, the location of principle points on the image plan are named intrinsic parameters). This issue is usually considered as a registration problem. Some authors use linear techniques (e.g., Faugeras and Toscani 1987), where least squares method is employed to estimate the intrinsic parameters and the pose (i.e., the position and the orientation of the calibration pattern frame in the sensor frame). Other techniques use

non-linear optimization methods (Brown, 1971). It consists in minimizing the error between the observation and the forward-projection of the model. In Tsai (1987) and Wei and Ma (1994), a linear estimation of some parameters 
is considered and the others are estimated iteratively. Alternatively, another technique (Ma et al., 2004), called self-calibration, do not use any calibration pattern but the parameters are estimated by moving a camera in a static scene. Constraints are provided by the scene rigidity in this approach.

Since the structure of a scanning electron microscope is very different from the structure of an optical microscope, it became apparent that novel image analysis, geometrical projection models and calibration processes would be necessary in order to extract accurate information from the SEM images. Postek et al. (1993) has demonstrated that accurate SEM calibration and error analysis was one of the major problems when considering such sensor. In earlier studies, photogrammetric analysis of SEM has been considered by some authors (Boyde, 1973; Ghosh, 1975). Several photogrammetric related calibration methods (Boyde, 1970; Wergin, 1985; Minnich et al., 1999) have been proposed for three dimensional imagery and reconstruction in SEM.

The projection model relates a 3D point on a specimen in the observed space to its projection in the $2 \mathrm{D}$ image. The perspective projection, where objects are projected towards a point (the center of projection), is used in classical camera models. The parallel projection (typically orthographic projection) corresponds to a perspective projection with an infinite focal length. The projection rays and the image plane is perpendicular in parallel projection model. It is noticed that this projection model is similar to the model used for telecentric lenses (Li and Tian, 2013)(Chen et al., 2014). In (Chen et al., 2014) a telecentric stereo micro-vision system is calibrated by solving 
a problem of sign ambiguity induced by the planar-object-based calibration technique. Previous studies on SEM consider that at low magnification, perspective projection model can be applied because the observed area and electron beam sweep angle are both large. At higher magnification, the center of projection is usually regarded at infinity so the parallel projection model is assumed. However, the practical limit between the choice of perspective projection and parallel projection model is not clear. Some experiments (Cornille et al., 2003; Sinram et al., 2002) show that parallel projection is assumed at magnification of $1000 \times$ and higher. Howell (1978) has concluded that the use of parallel projection depends on the desired accuracy for the calculation of position of a point on the specimen.

Another important issue in calibration is distortion, which contains spatial distortion (static distortion) and time-dependent drift (temporally-varying distortion). The drift is mainly due to presence of nonlinearities and instabilities during the raster scanning a specimen surface by the electron beam (Maune, 1976; Mizuno et al., 1997). This drift can be calibrated and be compensated as shown in (Cornille, 2005; Sutton et al., 2006; Malti et al., $2012 b)$. However, few authors have investigated the spatial distortion for an accurate calibration of SEM. One reason might be the complexity of modeling of distortions in a high magnification, where the common model of distortion is weakened. Several papers (Lacey et al., 1996; Sinram et al., 2002) ignore distortion and consider only a pure projection model. A few authors (Ghosh, 1975; Hemmleb and Albertz, 2000) consider the spatial dis- 
tortion with parametric models. Spatial distortion including radial distortion and spiral distortion are introduced in their geometric model. Schreier et al. (2004) proposed to use a priori distortion estimation technique in combination with bundle-adjustment (Brown, 1976; Triggs et al., 2000) for an accurate calibration of SEM. In Cornille et al. (2003), this distortion removal function is determined before the calibration stage. In this method, good guesses are required in the measurement to ensure the accuracy.

Furthermore, El Ghazali (1984) proposed system calibration for a SEM since the traditionally laboratory calibration is not convenient for complicate systems where the compensation and the deterioration effect between the different system components are not taken into account. Recently, a landmark-based 3D calibration strategy (Ritter et al., 2006) has been proposed considering a 3D micrometer-sized reference structure with the shape of a cascade slope-step pyramid. The manufacture of this special 3D reference structure is nevertheless an important and different issue. Eberle et al. (2015) has shown a micro chip that features a hexagonal arrangement of calibration structures. Since different scales of magnification are needed in some applications, Malti et al. (2012a) considers the modeling magnificationcontinuous parameters of the static distortion and the projection of the SEM. Zhu et al. (2011) has proposed a stereo-vision system under a SEM. The system has been calibrated using distortion-corrected images of a planar object and grid for various orientations (Sutton et al., 2009).

In this article, the mentioned calibration problems of SEM: projection 
models and spatial distortions are addressed. A novel approach of SEM calibration involving a full scale non-linear optimization is proposed. The extrinsic parameters (the pose) computation and SEM calibration are considered simultaneously. The formulation is simple and versatile. An iterative algorithm has been developed to acquire accurate results of calibration. This article is organized as follows. First, geometrical projection model is described in Section 2. Section 3 introduces the principle of calibration procedure. The experimental results at various magnifications that validate the approach are shown in Section 4.

\section{Considered projection models for a SEM}

In this article the geometrical calibration of the system projection model is focused on. The final objective of our work is visual servoing tasks for object positioning and manipulation using a SEM. Therefore for simplicity issues classical projection models is considered. Whereas such model as a clear physical meaning when considering optical devices, this is no longer the case with a SEM. Nevertheless, for the targeted applications, considering classical projection model has proved to be sufficient (Ghosh, 1975) (such assertion may no longer be true for, e.g., structure characterization). It is however important to determine the nature of the projection models to be considered (Hemmleb and Albertz, 2000; Howell, 1978): perspective or parallel models (see Figure 1). In this section, both the perspective and 
parallel projection models including modeling of the image distortion are discussed.

\subsection{Perspective projection}

Let ${ }^{c} \mathbf{X}=\left({ }^{c} X,{ }^{c} Y,{ }^{c} Z, 1\right)^{\top}$ be the homogeneous coordinates of a point on the observed object expressed in the sensor frame $\mathcal{F}_{c}$ (located on the projection center $) . \mathbf{x}=(x, y, 1)^{\top}$ is the homogeneous coordinates of its projection on the image plane expressed in normalized coordinate (i.e., in meter). It can be expressed by (Ma et al., 2004)

$$
\left\{\begin{array}{l}
x=\frac{{ }^{c} X}{{ }^{c} Z} \\
y=\frac{{ }^{c} Y}{{ }^{c} Z}
\end{array}\right.
$$

leading in the actual image coordinates expressed in pixel $\mathbf{x}_{p}=(u, v)$ on the image plane and given by

$$
\left\{\begin{array}{l}
u=u_{0}+p_{x} x \\
v=v_{0}+p_{y} y
\end{array}\right.
$$

where $p_{x}$ and $p_{y}$ represent the pixel/meter ratio and $u_{0}, v_{0}$ the principal point coordinates in the image plane. According to (1) and (2), the general 
expression of perspective projection is:

$$
\left[\begin{array}{l}
u \\
v \\
1
\end{array}\right]=\underbrace{\left[\begin{array}{ccc}
p_{x} & 0 & u_{0} \\
0 & p_{y} & v_{0} \\
0 & 0 & 1
\end{array}\right]}_{\mathbf{K}} \underbrace{\left[\begin{array}{cccc}
1 & 0 & 0 & 0 \\
0 & 1 & 0 & 0 \\
0 & 0 & 1 & 0
\end{array}\right]}_{\Pi}\left[\begin{array}{c}
{ }^{c} X \\
{ }^{c} Y \\
{ }^{c} Z \\
1
\end{array}\right] .
$$

For the camera calibration on perspective projection model, $p_{x}, p_{y}, u_{0}$ and $v_{0}$ are considered as intrinsic parameters. We rewrite (3) as:

$$
\mathbf{x}_{p}=\mathbf{K} \boldsymbol{\Pi}^{c} \mathbf{X}
$$

As already stated, for calibration issue, we consider a calibration pattern for which the position of some $3 \mathrm{D}$ features are known in a reference frame $\mathcal{F}_{w}$. Let us denote ${ }^{w} \mathbf{X}=\left({ }^{w} X,{ }^{w} Y,{ }^{w} Z, 1\right)^{\top}$ the coordinates of a feature expressed in $\mathcal{F}_{w}$. Its projection in the image plane (See Figure 2) is then given by

$$
\mathbf{x}_{p}=\mathbf{K} \Pi^{c} \mathbf{T}_{w}{ }^{w} \mathbf{X}
$$

${ }^{c} \mathbf{T}_{w}$ is an homogeneous matrix defined such that:

$$
{ }^{c} \mathbf{T}_{w}=\left(\begin{array}{cc}
{ }^{c} \mathbf{R}_{w} & { }^{c} \mathbf{t}_{w} \\
\mathbf{0}_{3 \times 1} & 1
\end{array}\right)
$$


where ${ }^{c} \mathbf{R}_{w}$ and ${ }^{c} \mathbf{t}_{w}$ are the rotation matrix and translation vector that define the position of the vision sensor in the calibration pattern frame (note that being a rotation matrix, ${ }^{c} \mathbf{R}_{w}$ should respect the orthogonality constraints). ${ }^{c} \mathbf{T}_{w}$ is referred as the intrinsic parameters or sensor pose.

\subsection{Parallel projection}

In parallel projection model, the projection rays are parallel. As mentioned previously, the projection center lies at infinite. The coordinates of a 2D point $\mathbf{x}=(x, y)$ corresponds to its $3 \mathrm{D}$ coordinates ${ }^{c} \mathbf{X}$ :

$$
\left\{\begin{array}{l}
x={ }^{c} X \\
y={ }^{c} Y
\end{array}\right.
$$

leading to its position expressed in pixel $\mathbf{x}_{p}=(u, v)$ in the digital image is

$$
\left\{\begin{array}{l}
u=p_{x} x \\
v=p_{y} y
\end{array}\right.
$$


According to (7) and (8), the general expression of parallel projection can be written as

$$
\left[\begin{array}{l}
u \\
v \\
1
\end{array}\right]=\underbrace{\left[\begin{array}{ccc}
p_{x} & 0 & 0 \\
0 & p_{y} & 0 \\
0 & 0 & 1
\end{array}\right]}_{\mathbf{K}_{\perp}} \underbrace{\left[\begin{array}{llll}
1 & 0 & 0 & 0 \\
0 & 1 & 0 & 0 \\
0 & 0 & 0 & 1
\end{array}\right]}_{\boldsymbol{\Pi}_{\perp}}\left[\begin{array}{c}
{ }^{c} X \\
{ }^{c} Y \\
{ }^{c} Z \\
1
\end{array}\right] .
$$

Since there is no longer principle point in parallel projection, only $p_{x}$ and $p_{y}$ are considered as the intrinsic parameters. As in the previous case, we can rewrite (9) as:

$$
\mathbf{x}_{p}=\mathbf{K}_{\perp} \boldsymbol{\Pi}_{\perp}{ }^{c} \mathbf{X}
$$

and if we consider the 3D coordinates of 3D features in the calibration reference frame $\mathcal{F}_{w}$ we have

$$
\mathbf{x}_{p}=\mathbf{K}_{\perp} \boldsymbol{\Pi}_{\perp}{ }^{c} \mathbf{T}_{w}{ }^{w} \mathbf{X}
$$

\section{$2.3 \quad$ Image distortion}

In classical models (Heikkila and Silven, 1997), the most commonly used spatial distortion is radial distortion. Instead of (2) in perspective model, the relation between the point position $\mathbf{x}$ and the coordinates in the image 
plane $\mathbf{x}_{p}$ in perspective projection is expressed by

$$
\left\{\begin{array}{l}
u=u_{0}+p_{x} x+\delta_{u} \\
v=v_{0}+p_{y} y+\delta_{v}
\end{array} .\right.
$$

The radial distortion can be approximated using

$$
\left\{\begin{array}{c}
\delta_{u}=\tilde{u}\left(k_{1} r^{2}+k_{2} r^{4}+\ldots\right) \\
\delta_{v}=\tilde{v}\left(k_{1} r^{2}+k_{2} r^{4}+\ldots\right)
\end{array}\right.
$$

where $r^{2}=\tilde{u}^{2}+\tilde{v}^{2}, \tilde{u}=u-u_{0}$ and $\tilde{v}=v-v_{0}$. Usually, to compensate for the radial distortion, one or two coefficients are enough. Considering the SEM geometry, it has to be noted that in SEM image such distortion appears to be very small. This should be validated by experiments.

Another issue that can be considered is the skewness between the $\mathrm{x}$ - and $\mathrm{y}$-axis. In this case:

$$
\left\{\begin{array}{l}
u=u_{0}+p_{x} x+\gamma y \\
v=v_{0}+p_{y} y
\end{array} .\right.
$$

Typically, $\gamma$ is null when the pixel in $\mathrm{x}$ - and $\mathrm{y}$-axis is exactly rectangular.

Repeated in Klemperer and Barnett (1971), the spiral distortion is caused by the spiral of the electrons within the microscope column. It is usually given by

$$
\left\{\begin{array}{c}
u=u_{0}+p_{x}\left(x+\delta_{x}\right) \\
v=v_{0}+p_{y}\left(y+\delta_{y}\right)
\end{array}\right.
$$


where $\delta_{x}=s_{1}\left(x^{2} y+y^{3}\right), \delta_{y}=s_{2}\left(x^{3}+x y^{2}\right), s_{1}$ and $s_{2}$ are spiral coefficients.

In the parallel model, the distortion models that replace (8) are similar but $u_{0}$ and $v_{0}$ equal to zero in equation (12), (14) and (15).

\section{Non-linear calibration process}

Calibration is an old research area that received much attention since the early 70's, first in the photogrammetry community (e.g., Brown 1971) then in the computer vision and robotics communities (e.g., Faugeras and Toscani 1987; Tsai 1987; Weng et al. 1992, etc.). Performing the calibration leads to the estimation of the intrinsic camera parameters (image center, focal length, distortion) but also, as a by-product, extrinsic camera parameters (i.e., the pose). Various techniques exist to achieve the calibration. Among these techniques, full-scale non-linear optimization techniques (introduced within the photogrammetry community, Brown 1971) have proved to be very efficient. They consist in minimizing the error between the observation and the back-projection of the model. Minimization is handled using numerical iterative algorithms such as Newton-Raphson or Levenberg-Marquartd.

\subsection{Single image calibration}

As stated the goal is to minimize the error between the points extracted from the image $\mathbf{x}_{p}^{*}$ and the projection of the model of the calibration pattern for given model parameters (both intrinsic parameters and pose) $\mathbf{x}_{p}(\mathbf{r}, \xi)$. 
Denoting $\xi$ the set of intrinsic parameters to be estimated and $\mathbf{r} \in \operatorname{se}(3)$ a minimal representation of ${ }^{c} \mathbf{T}_{w}\left(\mathbf{r}=\left({ }^{c} \mathbf{t}_{w}, \theta \mathbf{u}\right)^{\top}\right.$ where $\theta$ and $\mathbf{u}$ are the angle and the axis of the rotation ${ }^{c} \mathbf{R}_{w}$ ), the problem can be formulated as:

$$
(\widehat{\mathbf{r}}, \widehat{\xi})=\underset{\mathbf{r}, \xi}{\operatorname{argmin}} \sum_{i=1}^{N}\left({ }^{i} \mathbf{x}_{p}^{*}-{ }^{i} \mathbf{x}_{p}(\mathbf{r}, \xi)\right)^{2}
$$

where $N$ is the number of points used in the calibration process. For each point $i,{ }^{i} \mathbf{x}_{p}(\mathbf{r}, \xi)=\mathbf{K} \Pi{ }^{c} \mathbf{T}_{w}{ }^{w} \mathbf{X}_{i}$ (for the perspective projection model without considering distortion) and ${ }^{i} \mathbf{x}_{p}(\mathbf{r}, \xi)=\mathbf{K}_{\perp} \boldsymbol{\Pi}_{\perp}{ }^{c} \mathbf{T}_{w}{ }^{w} \mathbf{X}_{i}$ (for the parallel projection model without considering distortion). The solution of this problem relies on an iterative minimization process such as a Gauss-Newton or a Levenberg-Marquardt method.

Solving equation (16) consists in minimizing the cost function $E(\mathbf{r}, \xi)=$ $\|\mathbf{e}(\mathbf{r}, \xi)\|$ defined by:

$$
E(\mathbf{r}, \xi)=\mathbf{e}(\mathbf{r}, \xi)^{\top} \mathbf{e}(\mathbf{r}, \xi), \text { with } \quad \mathbf{e}(\mathbf{r}, \xi)=\mathbf{x}_{p}(\mathbf{r}, \xi)-\mathbf{x}_{p}^{*}
$$

where $\mathbf{x}_{p}(\mathbf{r}, \xi)=\left(\ldots,{ }^{i} \mathbf{x}_{p}(\mathbf{r}, \xi), \ldots\right)^{\top}$ and $\mathbf{x}_{p}^{*}=\left(\ldots,{ }^{i} \mathbf{x}^{*}, \ldots\right)^{\top}$ where ${ }^{i} \mathbf{x}_{p}(\mathbf{r}, \xi)$ is computed using equation (2) or (8). To simplify the notation, let us simply denote $\mathbf{e}=\mathbf{e}(\mathbf{r}, \xi)$.

To minimize this cost function, an exponential decrease of the projection error is specified:

$$
\dot{\mathrm{e}}=-\lambda \mathbf{e}
$$


where $\lambda$ is a proportional coefficient. In (18), $\dot{\mathbf{e}}$ can be simply computed from the time variation of $\dot{\mathbf{x}}_{p}$ which is given by:

$$
\dot{\mathbf{x}}_{p}=\frac{\partial \mathbf{x}_{p}}{\partial \mathbf{r}} \frac{d \mathbf{r}}{d t}+\frac{\partial \mathbf{x}_{p}}{\partial \xi} \frac{d \xi}{d t}
$$

where $\mathbf{r}$ represent the (virtual) sensor position along the minimization trajectory (translation and rotation), $\mathbf{v}=\frac{d \mathbf{r}}{d t}$ is the (virtual) sensor velocity during the minimization ${ }^{2}$. Rewrite (19):

$$
\dot{\mathbf{x}}_{p}=\mathbf{J}_{p} \mathbf{V}
$$

where $\mathbf{V}=\left[\begin{array}{c}\mathbf{v} \\ \dot{\xi}\end{array}\right]$. Matrix $\mathbf{J}_{p}$ is the image Jacobian, it is given by:

$$
\mathbf{J}_{p}=\left[\begin{array}{ll}
\frac{\partial \mathbf{x}_{p}}{\partial \mathbf{r}} & \frac{\partial \mathbf{x}_{p}}{\partial \xi}
\end{array}\right]
$$

Combining (20) and (18), V can be rewritten as follows:

$$
\mathbf{V}=-\lambda \mathbf{J}_{p}^{+}\left(\mathbf{x}_{p}(\mathbf{r}, \xi)-\mathbf{x}_{p}^{*}\right)
$$

where $\mathbf{J}_{p}^{+}$is the pseudo inverse of matrix $\mathbf{J}_{p}$ and $\mathbf{V}$ being the parameters increment computed at each iteration of this minimization process.

\footnotetext{
${ }^{2}$ note that this process is close to the visual servoing technique and has been used for pose estimation in Marchand and Chaumette (2002)
} 


\subsection{Multi-image calibration}

In practice, the intrinsic parameters are usually obtained by different viewpoints of the calibration pattern from the same camera. The optimization scheme then requires the computation of a set of positions of calibration pattern and a common set of intrinsic parameters. In that case the global error to be minimized is given by

$$
E=\sum_{i=1}^{n}\left(\mathbf{e}_{i}^{\top} \mathbf{e}_{i}\right)
$$

where $n$ is the number of images used in the calibration process and

$$
\mathbf{e}_{i}=\mathbf{x}_{p}\left(\mathbf{r}_{i}, \xi\right)-\mathbf{x}_{p}^{*}
$$

Let $\mathbf{x}_{p}^{i}$ be a set of images features extracted from the $i^{\text {th }}$ image. In multiimage calibration, (20) can be rewritten as:

$$
\left[\begin{array}{c}
\dot{\mathbf{x}}_{p}^{1} \\
\dot{\mathbf{x}}_{p}^{2} \\
\vdots \\
\dot{\mathbf{x}}_{p}^{n}
\end{array}\right]=\mathbf{J}_{p}\left[\begin{array}{c}
\mathbf{v}^{1} \\
\mathbf{v}^{2} \\
\vdots \\
\mathbf{v}^{n} \\
\dot{\xi}
\end{array}\right]
$$


with

$$
\mathbf{J}_{p}=\left[\begin{array}{ccccc}
\frac{\partial \mathbf{x}_{p}^{1}}{\partial \mathbf{r}} & 0 & \cdots & 0 & \frac{\partial \mathbf{x}_{p}^{1}}{\partial \xi} \\
0 & \frac{\partial \mathbf{x}_{p}^{2}}{\partial \mathbf{r}} & 0 & 0 & \frac{\partial \mathbf{x}_{p}^{2}}{\partial \xi} \\
\vdots & \vdots & \ddots & \vdots & \vdots \\
0 & \cdots & 0 & \frac{\partial \mathbf{x}_{p}^{n}}{\partial \mathbf{r}} & \frac{\partial \mathbf{x}_{p}^{n}}{\partial \xi}
\end{array}\right]
$$

\subsection{Nonlinear optimization}

In the nonlinear minimization process, the optimization algorithm is an important issue. The general idea of minimizing a nonlinear function is to successively update the parameters such that the value of cost function decreases at each iteration as specified by equation (18). The Gauss-Newton method is usually used in nonlinear optimization as presented in equation (22).

Particularly, the measured values are small in the SEM imaging (point coordinates are expressed in micrometer $(\mu \mathrm{m})$ and nanometer $(\mathrm{nm}))$. Several numerical problems are then induced into the optimization algorithms. For example, these tiny values causes rank deficiency of Jacobian matrix $\mathbf{J}_{p}$. This is why the Levenberg-Marquardt method is considered, which is numerically more efficient:

$$
\mathbf{V}=-\lambda\left(\mathbf{J}_{p}^{\top} \mathbf{J}_{p}+\mu \mathbf{I}\right)^{-1} \mathbf{J}_{p}^{\top} \mathbf{e}
$$

where $\mathbf{I}$ is a identity matrix and $\mu$ is a coefficient whose typical value ranges from 0.001 or 0.0001 . By modifying $\mu$, the algorithm is set to adapt the input data and to avoid numerical issues. 


\subsection{Jacobian}

In this article, the computation of $\frac{\partial \mathbf{x}_{p}}{\partial \mathbf{r}}$ and $\frac{\partial \mathbf{x}_{p}}{\partial \xi}$ in the Jacobian $\mathbf{J}_{p}$ is presented with the two specified projection models above.

The image Jacobian $\frac{\partial \mathbf{x}_{p}}{\partial \mathbf{r}}$ relates the motion of a point in the image to the (virtual) sensor motion, it could be expressed by:

$$
\frac{\partial \mathbf{x}_{p}}{\partial \mathbf{r}}=\left[\begin{array}{cc}
p_{x} & 0 \\
0 & p_{y}
\end{array}\right] \mathbf{L}
$$

where $\mathbf{L}=\frac{\partial \mathbf{x}}{\partial \mathbf{r}}$ is the Jacobian which relates the motion of the projection of a point on image plan (coordinates expressed in meter) to the (virtual) sensor motion.

\subsubsection{Perspective projection}

In the perspective projection model, the Jacobian $\mathbf{L}$ is given by Comport et al. (2006):

$$
\mathbf{L}=\left[\begin{array}{cccccc}
-\frac{1}{Z} & 0 & \frac{x}{Z} & x y & -\left(1+x^{2}\right) & y \\
0 & -\frac{1}{Z} & \frac{y}{Z} & 1+y^{2} & -x y & -x
\end{array}\right]
$$


From (2), without considering the distortion in the camera model, the deviation of image feature $\mathbf{x}_{p}$ by intrinsic parameters $\xi=\left(p_{x}, p_{y}, u_{0}, v_{0}\right)$ is:

$$
\frac{\partial \mathbf{x}_{p}}{\partial \xi}=\left[\begin{array}{cccc}
x & 0 & 1 & 0 \\
0 & y & 0 & 1
\end{array}\right]
$$

Considering one coefficient $k$ in radial distortion $\left(k_{1}\right.$ in (13)), the skew factor $\gamma$ and spiral coefficient $s_{1}, s_{2}$ as distortion parameters, the deviation of image feature $\mathbf{x}_{p}$ by intrinsic parameters $\xi=\left(p_{x}, p_{y}, u_{0}, v_{0}, k, \gamma, s_{1}, s_{2}\right)$ with distortion factors is:

$$
\frac{\partial \mathbf{x}_{p}}{\partial \xi}=\left[\begin{array}{cc}
x+s_{1}\left(x^{2} y+y^{3}\right) & 0 \\
0 & y+s_{2}\left(x^{3}+x y^{2}\right) \\
1-k\left(r^{2}+2 \tilde{u}^{2}\right) & -2 k \tilde{u} \tilde{v} \\
-2 k \tilde{u} \tilde{v} & 1-k\left(r^{2}+2 \tilde{v}^{2}\right) \\
\tilde{u} r^{2} & \tilde{v} r^{2} \\
y & 0 \\
p_{x}\left(x^{2} y+y^{3}\right) & 0 \\
0 & p_{y}\left(x^{3}+x y^{2}\right)
\end{array}\right]^{\top}
$$




\subsubsection{Parallel projection}

In the parallel projection model, the Jacobian is given by:

$$
\mathbf{L}_{\perp}=\left[\begin{array}{cccccc}
-1 & 0 & 0 & 0 & -Z & y \\
0 & -1 & 0 & Z & 0 & -x
\end{array}\right]
$$

Comparing with equation (29), it is evident that the motion along the $\mathrm{z}$-axis is not observable. Therefore the depth of the calibration pattern cannot be

recovered. The deviation $\frac{\partial \mathbf{x}_{p}}{\partial \xi}$ without distortion for $\xi=\left(p_{x}, p_{y}\right)$ is given from (8):

$$
\frac{\partial \mathbf{x}_{p}}{\partial \xi}=\left[\begin{array}{cc}
x & 0 \\
0 & y
\end{array}\right]
$$

With distortion, it is expressed with $\xi=\left(p_{x}, p_{y}, k, \gamma, s_{1}, s_{2}\right)$ :

$$
\frac{\partial \mathbf{x}_{p}}{\partial \xi}=\left[\begin{array}{cc}
x+s_{1}\left(x^{2} y+y^{3}\right) & 0 \\
0 & y+s_{2}\left(x^{3}+x y^{2}\right) \\
\tilde{u} r^{2} & \tilde{v} r^{2} \\
y & 0 \\
p_{x}\left(x^{2} y+y^{3}\right) & 0 \\
0 & p_{y}\left(x^{3}+x y^{2}\right)
\end{array}\right]^{\top}
$$




\section{Experimental results}

In the experiments, a Carl Zeiss AURIGA 60 SEM has been used to validate the developed calibration method. It provides a wide magnification ranges from $12 \times$ to $1,000,000 \times$. Within the SEM a 6 -DoF platform is available, including $360^{\circ}$ continuous rotation and tilt from $-15^{\circ}$ to $70^{\circ}$. Another SEM Carl Zeiss EVO LS 25 has also been employed in the experiments. The magnification of this SEM ranges from $5 \times$ to $1,000,000 \times$.

A multi-scale planar calibration pattern ${ }^{3}$ (see Figure 3) is used in the calibration procedure. It is a hierarchy of chessboard grids where size of each square are of $25 \mu \mathrm{m}, 10 \mu \mathrm{m}, 5 \mu \mathrm{m}, 2 \mu \mathrm{m}$ and $1 \mu \mathrm{m}$. Acquired image size is $1024 \times 768$ pixels. Several sets of calibration images (Figure 4 ) have been acquired within the SEM with different magnifications ranging $300 \times$ up to $10,000 \times$. The images from AURIGA 60 SEM have been acquired with a medium scan speed (3.3 $\mathrm{\mu s} /$ pixel) and a fast scan speed $(0.25 \mu \mathrm{s} /$ pixel $)$ respectively. The images from EVO LS 25 have been acquired with a medium scan speed (2.5 $\mathrm{ks} /$ pixel). Each group (with a given magnification) contents 7 to 9 images of the pattern acquired from various poses with rotation around z-axis ranging from $0^{\circ}$ to $40^{\circ}$, and tilt from $0^{\circ}$ to $8^{\circ}$.

The proposed calibration procedure has been implemented with the ViSP library (Marchand and Chaumette, 2005). Considering the chessboard shape of the calibration pattern, OpenCV chessboard corners detector has been

\footnotetext{
${ }^{3}$ fabricated at FEMTO-ST institute, France
} 
employed in order to obtain a precise localization of each corner. A linear algorithm has been considered to have a first approximation of the calibration parameters (Zhang, 2000). The proposed multi-image iterative non-linear minimization method for calibration, using both perspective and projection model, is then used. The intrinsic parameters are then computed by minimizing the residual error between the projection of the pattern for the current estimated pose and the observed one.

\subsection{Minimization process and algorithm behavior}

To illustrate the behavior and performance of the proposed algorithm, the calibration of the SEM using a parallel projection model and without adding any distortion parameters is considered here.

AURIGA 60 SEM has been employed in this experiment. The SEM magnification has been set to $2000 \times$, the size of each pattern square is of 5 $\mu \mathrm{m}$. Eight images of the calibration pattern have been acquired from eight poses with rotation from $0^{\circ}$ up to $20^{\circ}$ and tilt from $0^{\circ}$ up to $8^{\circ}$ and used in the calibration process. The gain $\lambda$ in equation (18) in the algorithm is set to 0.4 . Figure 5(a) shows the residual error computed at each iteration of the minimization process. The evolution of intrinsic parameters $p_{x}$ and $p_{y}$ is shown in Figure 5(b). The residual error and the intrinsic parameters converge quickly even thought the value is significant at the beginning. Only a few iterations less than 50 are required by the process. Figure 6 presents

the estimated set of extrinsic parameters (estimated sensor poses) during the 
minimization process. It can be noted that, as expected, motion along the z-axis is not observable using the parallel projection model (in equation (32), the elements in the third column of $\mathbf{L}_{\perp}$ which correspond to the Jacobian of translation on z-axis are indeed null).

\subsection{Projection models}

Another experiment aims to test two projection models that can be possibly considered for the calibration of a SEM. To compare the performance with different scales, four magnifications are considered: 500×, 1000×, 2000× and $5000 \times$. Note that it is suggested in the literature (Cornille et al., 2003; Sinram et al., 2002) that perspective projection can be applied for a magnification up to $1000 \times$ whereas parallel projection should be considered for higher magnification. The images from AURIGA 60 SEM are firstly used in this experiment. Table 1 shows the estimated calibrated intrinsic parameters $p_{x}, p_{y}, u_{0}$ and $v_{0}$, the estimated distance $Z_{1}$ between sensor and calibration pattern (for the first image) and the residual error $\|\mathbf{e}\|$ in pixel. In all the case the algorithm converges and the registration error is less than 0.5 pixel per point which correspond to the noise level in corner extraction. It is quite clear from the estimation of parameters $p_{x}$ and $p_{y}$ that, with the perspective projection model, intrinsic parameters are inconsistent. Nevertheless the ratio $p_{x} /\left(Z_{1} M\right)$ is almost constant (see Table 2) which confirms the fact that the difference between $p_{x}$ (or $p_{y}$ ) and object depth is not observable. This motivates the choice of the parallel projection model for future visual 
servoing experiments despite the fact that depth motion are not observable.

Table 2 shows the $p_{(x, y)} /\left(Z_{1} M\right)$ for perspective projection and $p_{(x, y)} / M$ for parallel projection. These factors are approximately a constant value in the two projection models.

A wide range of magnifications from $300 \times$ to $10,000 \times$ considering parallel projection model have been tested. The images are acquired by a medium scan speed (see Figure 4(a),(b)). Results are shown in Table 3. The intrinsic parameters of AURIGA 60 SEM through magnifications are shown in Figure 7. The ratio between the computed intrinsic parameters $p_{x}, p_{y}$ and magnification $M$ is almost constant: as expected a quasi linear relation exists between $p_{x}, p_{y}$ and magnification as shown in Figure 7. It as to be noted that the residual error $\|\mathbf{e}\|$ is slightly more important for low magnification meaning that parallel projection model is less appropriate at low magnification $(300 \times, 500 \times)$ which confirms earlier report (Sinram et al., 2002). $\|\mathbf{e}\|$ also increases at high magnification but the reason is that at low magnification the extraction of corner position on the calibration pattern used in this experiment is far more accurate than that at high magnification.

To compare the performance of the proposed calibration process within different conditions, another set of images is acquired using fast scan speed (see Figure 4(c),(d) ). Results are shown in Table 4. It can be seen that the calibration results keep stable while the scan speed has been changed. From the results, $\|\mathbf{e}\|$ increases using fast scan speed due to the noise introduced into the images. 
Table 5 shows the calibration results on parallel projection from EVO LS 25 SEM. It can be noticed from the table the ratio between $p_{x}, p_{y}$ and $M$ is also almost constant as that in Table 3. Since the calibration images are acquired from different SEMs respectively, $p_{x} / M$ and $p_{y} / M$ are different from that in Table 3. Figure 8 shows the intrinsic parameters of EVO LS 25 SEM with respect to magnifications.

\subsection{Distortion issues}

Finally, an experiment has been realized to test the potential effects of distortion using AURIGA 60 SEM. Three magnifications are considered in this experiments: $500 \times, 2000 \times$ and $5000 \times$. To compare the performance of calibration with and without distortion parameters, all the factors (gain, coefficient in Levenberg-Marquardt optimization, etc.) in the algorithm are fixed. Table 6 shows the calibrated radial distortion parameter $k$, skewness parameter $\gamma$, intrinsic parameters $p_{x}^{\prime}, p_{y}^{\prime}$, residual error $\left\|\mathbf{e}^{\prime}\right\|$ with distortion and intrinsic parameters $p_{x}, p_{y}$, residual error $\|\mathbf{e}\|$ without distortion. Results are obtained on parallel projection model. It is evident that introducing distortion parameters does not affect the computation of the main intrinsic parameters $p_{x}, p_{y}$ and does not improve the residual error. In this case, such spatial distortion could be typically ignored in the calibration process. 


\section{Conclusions}

In this article, a simple and efficient method of SEM calibration has been addressed. A global multi-image non-linear minimization process that minimizes the residual error between the projection of the calibration pattern and its observation in the image was considered. The precise intrinsic parameters as well as the position of the sensor with respect to the pattern are computed. Since the lack of observation of the depth information of SEM image, the choice of the parallel projection model has been validated for SEM images. The spatial distortion parameters (skewness, radial distortion and spiral distortion) are such insignificant in the experiments that can be eliminated. 


\section{References}

A. Boyde. Practical problems and methods in the three-dimensional analysis of scanning electron microscope images. Scanning electron microscopy, 1970:105-112, 1970.

A. Boyde. Quantitative photogrammetric analysis and qualitative stereoscopic analysis of sem images. Journal of Microscopy, 98(3):452-471, 1973. ISSN 1365-2818.

D. C. Brown. The bundle adjustment-progress and prospects. Int. Archives Photogrammetry, 21(3):1-1, 1976.

D.C. Brown. Close-range camera calibration. Photogrammetric Engineering, 4(2):127-140, March 1971 .

Z. Chen, H. Liao, and X. Zhang. Telecentric stereo micro-vision system: Calibration method and experiments. Optics and Lasers in Engineering, 57:82-92, June 2014.

A.I. Comport, E. Marchand, M. Pressigout, and F. Chaumette. Real-time markerless tracking for augmented reality: the virtual visual servoing framework. IEEE Trans. on Visualization and Computer Graphics, 12 (4):615-628, July 2006.

N. Cornille. Accurate 3D shape and displacement measurement using a scan- 
ning electron microscope. PhD thesis, École des Mines d'Albi, France, 2005.

N. Cornille, D. Garcia, M. A. Sutton, S. McNeill, and J.-J. Orteu. Automated 3-d reconstruction using a scanning electron microscope. In SEM annual conf. ES exp. on experimental and applied mechanics, 2003.

A.L. Eberle, S Mikula, R Schalek, J.W. Lichtman, M.L. Tate, and D Zeidler. High-resolution, high-throughput imaging with a multibeam scanning electron microscope. Journal of microscopy, 2015.

M. El Ghazali. System calibration of scanning electron microscopes. International Archives of Photogrammetry and Remote Sensing, 25:258-266, 1984.

O.D Faugeras and G. Toscani. Camera calibration for 3D computer vision. In Proc Int. Workshop on Machine Vision and Machine Intelligence, pages 240-247, Tokyo, February 1987.

S. K. Ghosh. Photogrammetric calibration of a scanning electron microscope. Photogrammetria, 31(3):91 - 114, 1975.

J. Heikkila and O. Silven. A four-step camera calibration procedure with implicit image correction. In Proceedings of the IEEE Computer Society Conference Computer Vision and Pattern Recognition, pages 1106-1112, 1997. 
M. Hemmleb and J. Albertz. Microtopography-the photogrammetric determination of friction surfaces. International Archives of Photogrammetry and Remote, 33:56-63, 2000.

P. G. T. Howell. A theoretical approach to the errors in sem photogrammetry. Scanning, 1(2):118-124, 1978.

O. Klemperer and M. E. Barnett. Electron optics. Cambridge University Press, Cambridge, U.K., 1971.

A. J. Lacey, N. A. Thacker, and R. B. Yates. Surface approximation from industrial sem images. In British Machine Vision Conference, pages 725$734,1996$.

D. Li and J. Tian. An accurate calibration method for a camera with telecentric lenses. Optics and Lasers in Engineering, 51(5):538 - 541, 2013.

Y. Ma, S. Soatto, J. Košecká, and S. Sastry. An invitation to 3-D vision. Springer, 2004.

A. C. Malti, S. Dembélé, N. Le Fort-Piat, P. Rougeot, and R. Salut. Magnification-continuous static calibration model of a scanning-electron microscope. Journal of Electronic Imaging, 21(3):033020-1, 2012a.

A. C. Malti, S. Dembélé, N. Le Piat, C. Arnoult, and N. Marturi. Toward fast calibration of global drift in scanning electron microscopes with respect to time and magnification. International Journal of Optomechatronics, 6(1): $1-16,2012 b$. 
E. Marchand and F. Chaumette. Virtual visual servoing: a framework for real-time augmented reality. In G. Drettakis and H.-P. Seidel, editors, EUROGRAPHICS'02 Conf. Proceeding, volume 21(3) of Computer Graphics Forum, pages 289-298, Saarebrücken, Germany, September 2002.

E. Marchand and F. Chaumette. Feature tracking for visual servoing purposes. Robotics and Autonomous Systems, 52(1):53-70, June 2005. special issue on "Advances in Robot Vision", D. Kragic, H. Christensen (Eds.).

D. F. Maune. Photogrammetric self-calibration of scanning electron microscopes. Photogrammetric Engineering and Remote Sensing, 42(9), 1976.

B. Minnich, H. Leeb, E. Bernroider, and A. Lametschwandtner. Threedimensional morphometry in scanning electron microscopy: a technique for accurate dimensional and angular measurements of microstructures using stereopaired digitized images and digital image analysis. Journal of Microscopy, 195(1):23-33, 1999.

F. Mizuno, M. Shimizu, K. Sasada, and T. Mizuno. Evaluation of the longterm stability of critical-dimension measurement scanning electron microscopes using a calibration standard. Journal of Vacuum Science \& Technology B, 15(6):2177-2180, 1997.

M.T. Postek, A.E. Vladar, S.N. Jones, and W.J. Keery. Interlaboratory study on the lithographically produced scanning electron microscope magnifica- 
tion standard prototype. Journal of research of the National Institute of Standards and Technology, 98:447-447, 1993.

M. Ritter, M. Hemmleb, B. Lich, P. Faber, and H. Hohenberg. Sem/fib stage calibration with photogrammetric methods. In ISPRS Commission V Symp. 2006 (Int. Archives of Photogrammetry, Remote Sensing and Spatial Information Sciences), volume 36, 2006.

H. W. Schreier, D. Garcia, and M. A. Sutton. Advances in light microscope stereo vision. Experimental mechanics, 44(3):278-288, 2004.

O. Sinram, M. Ritter, S. Kleindick, A. Schertel, H. Hohenberg, and J. Albertz. Calibration of an sem, using a nano positioning tilting table and a microscopic calibration pyramid. International Archives of the Photogrammetry, Remote Sensing and Spatial Information Sciences, 34(5):210-215, 2002.

M. A. Sutton, N. Li, D. Garcia, N. Cornille, J.-J. Orteu, S. R. McNeill, H. W. Schreier, and X. Li. Metrology in a scanning electron microscope: theoretical developments and experimental validation. Measurement Science and Technology, 17(10):2613, 2006.

M.A. Sutton, J.-J. Orteu, and H Schreier. Image Correlation for Shape, Motion and Deformation Measurements: Basic Concepts, Theory and Applications. Springer Publishing Company, Incorporated, 1st edition, 2009. 
B. Triggs, P. F. McLauchlan, R. I. Hartley, and A. W. Fitzgibbon. Bundle adjustment - a modern synthesis. In Vision algorithms: theory and practice, pages 298-372. Springer, 2000.

R.Y. Tsai. A versatile camera calibration technique for high-accuracy 3D machine vision metrology using off-the-shelf tv cameras and lenses. IEEE Journal of Robotics and Automation, 3(4):323-344, August 1987.

G.-Q. Wei and S.D. Ma. Implicit and explicit camera calibration: Theory and experiments. IEEE Trans. on Pattern Analysis and Machine intelligence, 16(5):469-480, May 1994.

J. Weng, P. Cohen, and N. Rebiho. Motion and structure estimation from stereo image sequences. IEEE Trans. on Robotics and Automation, 8(3): 362-382, June 1992.

W. Wergin. Three-dimensional imagery and quantitative analysis in sem studies of nematodes. Agriculture, ecosystems $\& 3$ environment, 12(4):317334,1985 .

Z. Zhang. A flexible new technique for camera calibration. IEEE Trans. on Pattern Analysis and Machine Intelligence, 22(11):1330-1334, November 2000.

T. Zhu, M.A. Sutton, N. Li, J.-J. Orteu, N. Cornille, X. Li, and A.P. Reynolds. Quantitative stereovision in a scanning electron microscope. Experimental Mechanics, 51(1):97-109, 2011. 